\title{
Cirurgias Êntero-Colorretais - Abordagem Cirúrgica de 129 Pacientes do SUS no Programa de Pós-Graduação Sensu Lato em Coloproctologia
}

\author{
Abdominal Entero-colorectal Surgery - Surgical Approach of 129 Patients \\ from a Public Health Service from a Post Graduate Program (Residency) \\ in Coloproctology
}

\author{
RODRIGO GUIMARÃES OLIVEIRA' ${ }^{1}$, FLAVIAFONTES FARIA ${ }^{1}$, ANTONIO CARLOS BARROS LIMAJUNIOR ${ }^{1}$, \\ FABIO GONTIJO RODRIGUES ${ }^{1}$, MÔNICAMOURTHÉ DE ALVIM ANDRADE ${ }^{2}$, DANIEL MARTINS BARBOSA \\ MEDEIROS GOMES ${ }^{2}$, PETERSON MARTINS NEVES ${ }^{3}$, JOSÉ ROBERTO MONTEIRO CONSTANTINO ${ }^{3}$, ÁUREACÁSSIA \\ GUALBETO BRAGA ${ }^{3}$, RENATA MAGALISILLUZIO FERREIRA ${ }^{3}$, ISABELLAMENDONÇAALVARENGA ${ }^{3}$, \\ DAVID DE LANNA ${ }^{3}$, RICARDO GUIMARÃES TEIXEIRA ${ }^{3}$, HERALDO NEVES VALLE JUNIOR ${ }^{3}$, SINARAMÔNICA \\ OLIVEIRALEITE ${ }^{3}$, LUCIANAMARIAPYRAMO COSTA ${ }^{3}$, ILSON GERALDO DA SILVA $^{4}$, GERALDO MAGELA \\ GOMESDACRUZ
}

\author{
${ }^{1}$ Residentes; ${ }^{2}$ Assistentes voluntários; ${ }^{3}$ Assistentes efetivos; ${ }^{4}$ Chefe interino do Serviço de Coloproctologia de Homens \\ da Santa Casa de Belo Horizonte; Chefe do Serviço de Coloproctologia de Mulheres e do Ambulatório de \\ Coloproctologia da Santa Casa de Belo Horizonte e Coordenador do Grupo de Coloproctologia da Santa Casa de Belo \\ Horizonte e Faculdade de Ciências Médicas de Minas Gerais.
}

\begin{abstract}
OLIVEIRA RG; FARIA FF; LIMA JUNIOR ACB; RODRIGUES FG; ANDRADE MMA; GOMES DMBM; NEVES PM; CONSTANTINO JRM; BRAGA ACG; FERREIRA RMS; ALVARENGA IM; LANNA D; TEIXEIRA RG; VALLE JUNIOR HN; LEITE SMO; COSTA LMP; SILVA IG; CRUZ GMG. Cirurgias Êntero-Colorretais - Abordagem Cirúrgica de 129 Pacientes do SUS no Programa de Pós-Graduação Sensu Lato em Coloproctologia. Rev bras Coloproct, 2010;30(3): 333-343.
\end{abstract}

RESUMO: Dentro do Programa de pós-graduação em Coloproctologia, durante o ano 2009, os dois pós-graduandos de segundo ano realizaram, como cirurgiões principais, 129 cirurgias de grande porte, sempre assistidos, efetivamente, por um ou dois preceptores. Todas as cirurgias foram realizadas em pacientes do SUS, na Santa Casa de Belo Horizonte, com absoluta presença dos membros do Grupo de Coloproctologia da Santa Casa e Faculdade de Ciências Médicas de Minas Gerais (GCP-SCBH-FCMMG). Foi feita uma análise retrospectiva dos 129 prontuários, permitindo várias observações importantes. A média etária dos pacientes foi 56,9 anos, com extremos de 25 e 87 anos, sendo as sexta e sétima décadas a mais representativa, respectivamente com $25,6 \%$ e $24,8 \%$, totalizando $50,4 \%$ dos 55 pacientes (p<0,05). Dos 129 pacientes, $51,2 \%$ eram sexo feminino $(\mathbf{5 1 , 2 \%})(\mathrm{p}>\mathbf{0 , 0 5})$. A entidade nosológica mais comum foi o câncer colorretal (74 casos; $57,4 \%)$, seguindo as ileostomias (16 casos; $12,4 \%$ ) e as complicações cirúrgicas (11 casos; 8,5\%). As cirurgias mais realizadas foram as retossigmoidectomia com anastomose colorretal ( 35 casos; $27,1 \%$ ), as hemicolectomias direitas com anastomose ileotransverso ( 20 casos; $15,5 \%$ ) e o restabelecimento de trânsito intestinal de ileostomia (16 casos;12,4\%). Das 129 cirurgias $53(41,1 \%)$ não envolveram anastomoses e $76(58,9 \%)$ envolveram ressecções intestinais e anastomoses. Houve oito comorbidades $(6,2 \%)$, sendo a caquexia (três casos) a mais comum. Houve 17 complicações $(13,2 \%), 11$ envolvendo as 76 ressecções com anastomose $(\mathbf{1 4 , 5 \%})$ e seis as ressecções sem anastomoses $(\mathbf{1 1 , 3 \%})$. As complicações mais comuns entre as 11 provenientes de ressecções e anastomoses foram as deiscências (sete; 9,2\%). As anastomoses mecânicas (55) complicaram mais $(16,3 \%)$ que as manuais $(21)(9,5 \%)$. Houve 14 óbitos $(10,8 \%)$, sendo seis $(4,6 \%)$ devidos à sepse, quatro $(3,1 \%)$ devido a TEP e quatro $(3,1 \%)$ devido a falência múltipla de órgãos. Dos 14 óbitos, quatro $(3,1 \%)$ foram decorrentes de complicações cirúrgicas e dez $(\mathbf{7 , 7 \%})$ foram decorrentes de co-morbidades.

Descritores: Cirurgia colorretal; cirurgia abdominal; estudo retrospectivo; SUS; pós-graduação.

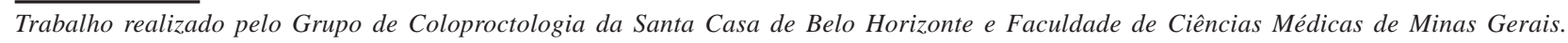

Recebido em 09/06/2010

Aceito para publicação em 30/07/2010 
Rev bras Coloproct Julho/Setembro, 2010
Cirurgias Êntero-Colorretais - Abordagem Cirúrgica de 129 Pacientes do SUS no Programa de Pós-Graduação Sensu Lato em Coloproctologia Rodrigo Guimarães Oliveira e Cols.
Vol. 30

\section{INTRODUÇÃO}

Dentro do Programa de pós-graduação em Coloproctologia, durante o ano 2009, os dois pósgraduandos de segundo ano realizaram, como cirurgiões principais, 129 cirurgias de grande porte. Todas as cirurgias foram realizadas em pacientes do SUS, na Santa Casa de Belo Horizonte, com absoluta presença dos membros do Grupo de Coloproctologia da Santa Casa e Faculdade de Ciências Médicas de Minas Gerais (GCP-SCBH-FCMMG).

\section{OBJETIVO}

O objetivo do trabalho foi proceder a uma revisão criteriosa de uma casuística de 11 meses (2009) de 129 pacientes operados de várias doenças ênterocolorretais, submetidos a algum tipo de ressecção e anastomose, sendo todos os pacientes do SUS e operados pelo Residente R2, sempre auxiliado por um ou dois preceptores.

\section{CASUÍSTICA - MATERIAL E MÉTODO}

Dentro do Programa de pós-graduação em Coloproctologia, durante o ano 2009, os dois pósgraduandos de segundo ano realizaram, como cirurgiões principais, 129 cirurgias de grande porte, sempre assistidos, efetivamente, por um ou dois preceptores. Todas as cirurgias foram realizadas em pacientes do SUS, na Santa Casa de Belo Horizonte, com absoluta presença dos membros do Grupo de Coloproctologia da Santa Casa e Faculdade de Ciências Médicas de Minas Gerais (GCP-SCBH-FCMMG).

Os prontuários dos pacientes foram minuciosamente revisados, dando-se absoluta atenção aos seguintes dados: idade, gênero, nome do cirurgião principal (residente R2), auxiliares (preceptores que ajudaram a cirurgia), diagnóstico, resultado da colonoscopia (e biópsia, quando houve), resultado do exame histopatológico das biópsias, preparo intestinal do paciente para a cirurgia (se houve e com que substância), o tempo de cirurgia, a técnica cirúrgica usada, a forma de anastomose executada (em que lugar anatômico do cólon e/ou reto e com que artefato, se manual ou mecânica), o bisturi e os grampeadores usados na cirurgia, tipo e extensão da incisão abdominal, intercorrências peroperatórias, dimensões da peça cirúrgica, complicações pós-operatórias de natureza cirúrgica e de na- tureza sistêmica, a abordagem das complicações pósoperatórias, o período de internamento hospitalar, o período de permanência em CTI, dia de liberação da dieta, o exame histopatológico da peça cirúrgica e as co-morbidades apresentadas pelos pacientes. Foi, da mesma forma, feita uma correlação entre morbimortalidade e co-morbidade, uma vez que os pacientes, em marcante número de vezes, apresentavam estado geral precário e acumulando outras doenças além do tumor colorretal.

Tabela 1 - Distribuição, por décadas etárias, de 129 pacientes submetidos a cirurgias ênterocolorretais, por dois médicos residentes em Coloproctologia como cirurgiões principais, no Programa de Pós-Graduação "sensu lato" do Grupo de Coloproctologia da SCBH e FCMMG.

\begin{tabular}{c|c|c|c|c}
\hline Idades (anos) & $\mathbf{N}$ & $\mathbf{\%}$ & $\mathbf{S ~ N}$ & $\mathbf{S} \%$ \\
\hline$<10$ & 0 & 0 & 0 & 0 \\
\hline $11-20$ & 0 & 0 & 0 & 0 \\
\hline $21-30$ & 3 & 2,3 & 3 & 2,3 \\
\hline $31-40$ & 15 & 11,6 & 18 & 13,9 \\
\hline $41-50$ & 22 & 17,1 & 40 & 31,0 \\
\hline $\mathbf{5 1 - 6 0 *}$ & $\mathbf{3 3}$ & 25,6 & $\mathbf{7 3}$ & 56.6 \\
\hline $\mathbf{6 1 - 7 0 *}$ & $\mathbf{3 2}$ & 24,8 & 105 & 81,4 \\
\hline $71-80^{*}$ & 18 & 14,0 & 123 & 95,4 \\
\hline $81-90$ & 6 & 4,6 & 129 & 100,0 \\
\hline $91-100$ & 0 & 0 & 129 & \\
\hline Total & $\mathbf{1 2 9}$ & $\mathbf{1 0 0 , 0}$ & 129 & 100,0 \\
\hline
\end{tabular}

Média: 56,9 anos; Mínima: 25 anos; Máxima: 87 anos; 51-60 anos: 33 (25,6); 61-70 anos: 32 (24,8); Faixas etárias com $p<0,05$.

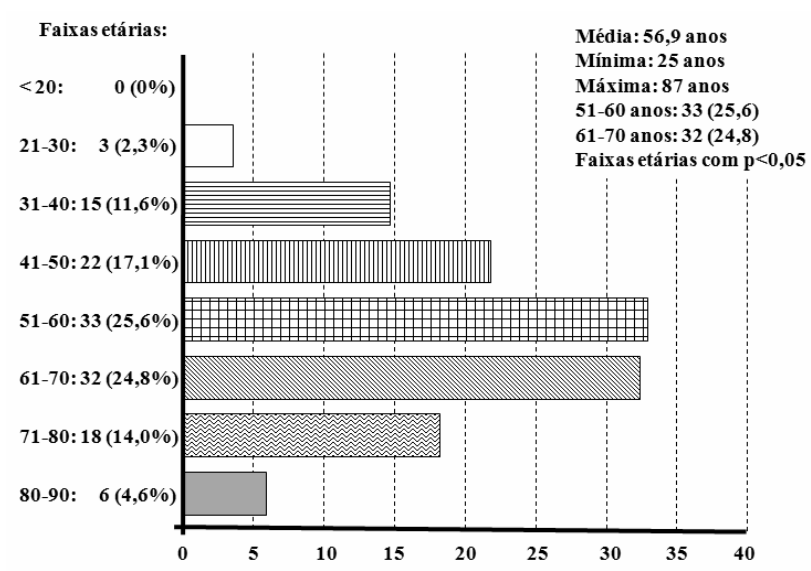

Figura 1 - Distribuição, por décadas etárias, de 129 pacientes submetidos a cirurgias êntero-colorretais, por dois médicos residentes em Coloproctologia como cirurgiões principais, no Programa de Pós-Graduação "sensu lato" do Grupo de Coloproctologia da SCBH e FCMMG. 
Cirurgias Êntero-Colorretais - Abordagem Cirúrgica de 129 Pacientes do SUS no Programa de Pós-Graduação Sensu Lato em Coloproctologia Rodrigo Guimarães Oliveira e Cols.
Vol. 30

\section{RESULTADOS}

Idade e gênero: A média etária dos pacientes foi 56,9 anos, com extremos de 25 e 87 anos, sendo as sexta e sétima décadas a mais representativa, respectivamente com 33 pacientes $(25,6 \%)$ e 32 pacientes $(24,8 \%)$, totalizando 55 pacientes $(50,4 \%)$ (tabela 1 e figura 1) $(\mathrm{p}<0,05)$. Quanto ao gênero, 66 pacientes eram do sexo feminino $(51,2 \%)$ e 63 do sexo masculino $(48,8)$ (tabela 2 e figura 2$)(\mathrm{p}>0,05)$.

Diagnósticos e objetivos das cirurgias: Das 129 cirurgias realizadas a maioria dos casos ( 74 casos; $57,4 \%$ ) foi para abordar câncer colorretal, dos quais, 23 casos de câncer de sigmóide, 26 de câncer no reto, 13 de câncer no ceco, sete de câncer de outras localizações colorretais e quatro de recidiva pélvica de câncer colorretal. Os demais 55 casos $(42,6 \%)$ foram para abordar ileostomias (16 casos; 12,4\%), complicações cirúrgicas (11 casos; 8,5\%), pólipos colorretais (seis casos; 4,6\%) e outras condições colorretais (22 casos; $17,1 \%)$. As 11 complicações envolveram deiscências de anastomoses (três casos), fístulas anastomóticas (três casos), estenoses anastomóticas (dois casos), evisceração (dois casos) e abscesso peri-anastomótico

Tabela 2 - Distribuição, por gênero dos pacientes, de 129 pacientes submetidos a cirurgias ênterocolorretais, por dois médicos residentes em Coloproctologia como cirurgiões principais, no Programa de Pós-Graduação "sensu lato" do Grupo de Coloproctologia da Santa Casa de Belo Horizonte.

\begin{tabular}{l|c|c|}
\hline \multicolumn{1}{|c|}{ Gêneros } & N & \% \\
\hline Feminino & 66 & 51,2 \\
\hline Masculino & 63 & 48,8 \\
\hline Total & 129 & 100,0 \\
\hline
\end{tabular}

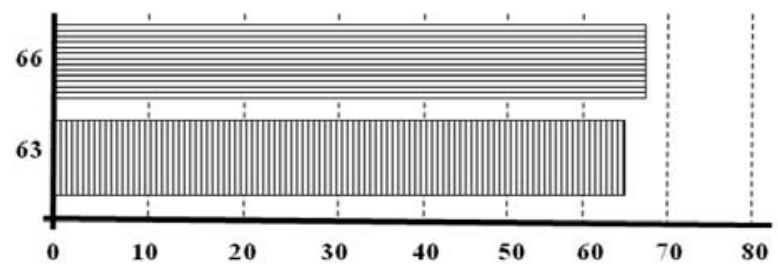

Figura 2 - Distribuição, por gênero dos pacientes, de 129 pacientes submetidos a cirurgias êntero-colorretais, por dois médicos residentes em Coloproctologia como cirurgiões principais, no Programa de Pós-Graduação "sensu lato" do Grupo de Coloproctologia da Santa Casa de Belo Horizonte. (um caso). Dos seis casos de pólipos colorretais, três se localizavam no sigmóide, um no ceco um no cólon transverso e um no reto. As outras condições colorretais mórbidas incluiam: doeça de Crohn (quatro casos), enterite e retite actínica com fístula (quatro casos), portadores de colostomia por cirurgia de Hartmann anterior, procidência retal (dois casos), megacólon chagásico (dois casos), endometriose de sigmóide (dois casos), além de um caso de abdome agudo inflamatório por apendicite, abdome agudo perfurativo por doença diverticular de sigmóide, hidradenite supurativa perineal, diverticulose colônica difusa e fístula de bolsa ileal (tabela 3 e figura 3).

Cirurgias realizadas: Foram as seguintes as 129 cirurgias realizadas (tabela 4 e figura 4): retossigmoidectomia com anastomose colorretal (35 casos; $27,1 \%$ ), hemicolectomia direita com anastomose ileo-transverso (20 casos; $15,5 \%$ ), restabelecimento de trânsito intestinal de ileostomia (16 casos;12,4\%), laparotomia com colostomia (10 casos; $7,8 \%$ ), cirurgia de Hartmann (sete casos; $5,4 \%$ ), colectomia total com anastomose ileo-retal $(6$ casos; $4,7 \%)$, l'parotomia com ileostomia (seis casos;4,7\%), retossigmoidectomia abdominal com anastomose colorretal por duplo grampeamento (cinco casos; 3,8\%), cirurgia de Duhamell (cinco casos; $3,8 \%$ ), hemicolectomia esquerda com anastomose transverso-retal (dois casos; 1,6\%), reabaixamento colorretal (dois casos; $1,6 \%$ ), amputação abdominoperineal com colostomia terminal (dois casos $; 1,6 \%$ ), enterectomia (dois casos; $1,6 \%$ ), proctocolectomia total com ileostomia terminal (dois casos; $1,5 \%$ ), laparorrafia (dois casos; $1,6 \%$ ), ressecção transanal (dois casos; $1,6 \%$ ), enterectomia e panhisterectomia (dois casos; $1,6 \%$ ), reanastomose (um caso; $0,8 \%$ ), retossigmoidectomia perineal (um caso; $9,8 \%$ ) e enterectomia com lise de aderências (um caso; $0,8 \%$ ) (tabela 4 e figura 4).

Das 129 cirurgias realizadas $53(41,1 \%)$ não envolveram anastomoses e $76(58,9 \%)$ envolveram ressecções intestinais e anastomoses (tabela 5 e figuras 5 e 6). E foram as seguintes as cirurgias envolvendo anastomoses êntero-colorretais: retossigmoidectomia com anastomose colorretal (35 casos; 46,1\%), hemicolectomia direita com anastomose ileo-transverso (20 casos; $26,3 \%)$, colectomia total com anastomose ileo-retal (cinco casos; 6,6\%), cirurgia de Duhamell (cinco casos;6,6\%), hemicolectomia esquerda com anastomose transverso-retal (dois casos; 2,6\%), 
Rev bras Coloproct Julho/Setembro, 2010
Cirurgias Êntero-Colorretais - Abordagem Cirúrgica de 129 Pacientes do SUS no Programa de Pós-Graduação Sensu Lato em Coloproctologia Rodrigo Guimarães Oliveira e Cols.
Vol. 30 $\mathbf{N}^{\circ} 3$

Tabela 3 - Distribuição, por diagnósticos principais, de 129 pacientes submetidos a cirurgias ênterocolorretais, por dois médicos residentes em Coloproctologia como cirurgiões principais, no Programa de Pós-Graduação "sensu lato" do Grupo de Coloproctologia da SCBH e FCMMG.

\begin{tabular}{|r|r|c|}
\hline DIAGNÓSTICOS PRINCIPAIS & \multicolumn{1}{c|}{ N } & \multicolumn{1}{c|}{$\%$} \\
\hline CÂNCER COLORRETAL & 74 & 57,4 \\
\hline Câncer de sigmóide & 23 & \\
\hline Câncer de reto & 26 & \\
\hline Câncer de ceco & 13 & \\
\hline Outras localizaçes & 7 & \\
\hline Recidiva pélvica de câncer de reto & \multicolumn{1}{|c|}{16} & 12,4 \\
\hline ILEOSTOMIA & 11 & 8,5 \\
\hline COMPLICAÇÕES CIRÚRGICAS OPERADAS & 6 & \\
\hline Deiscências (3) e fístulas (3) de anastomoses & 2 & \\
\hline Estenose anastomose & 2 & \\
\hline Evisceração & 1 & \\
\hline Abscesso peri-anastomose & 06 & 4,6 \\
\hline PÓLIPOS SIGMOIDE (3), CECO (1), TRANSVERSO (1), RETO (1) & 22 & 17,1 \\
\hline OUTRAS CONDIÇõES MÓRBIDAS COLORRETAIS & 4 & \\
\hline DC ileal, DC cólon c/ Ft e DC reto c/ Ft & 4 & \\
\hline Enterite e retite actínica com Ft & 3 & \\
\hline Hartmann & 2 & \\
\hline Procidência retal & 2 & \\
\hline Megacólon Chagásico & 2 & \\
\hline Endometriose de sigmóide & 1 & \\
\hline Abdome agudo inflamatório (apendicite) & 1 & \\
\hline AAP por doença diverticular de sigmóide & 1 & \\
\hline Hidradenite & 1 & \\
\hline Diverticulose colônica difusa & 129 & 100 \\
\hline Fístula de bolsa ileal &
\end{tabular}

reabaixamento colorretal (dois casos; $2,6 \%$ ), reanastomose (um caso; $1,3 \%$ ) (tabela 5 e figuras 5 e 6 ).

Co-morbidades e Morbimortalidade: Houve oito co-morbidades: caquexia (três casos), hipertensão arterial sistêmica (dois casos), obesidade (um caso), diabete e anemia (um caso), bronquiectasia (um caso) (tabelas 6, 7 e 8 e figura 7). Houve 17 complicações $(13,2 \%)$ assim distribuídas: as 11 complicações envolvendo as 76 ressecções com anastomose $(14,5 \%)$ ocorreram nas 35 RSA-ACR (três deiscências de anastomose e uma fístula retovaginal), nas $20 \mathrm{HCD}$ AIT (duas deiscências de anastomose e uma TEP), nas seis CT-AIR (uma deiscência de anastomose, um abscesso perianastomótico e uma trombose mesentérica) e nos 5 RSA-ACR DuGr (uma deiscência de anastomose (tabela 8 e figura 9). As complicações decorrentes de anastomoses mecânicas (55

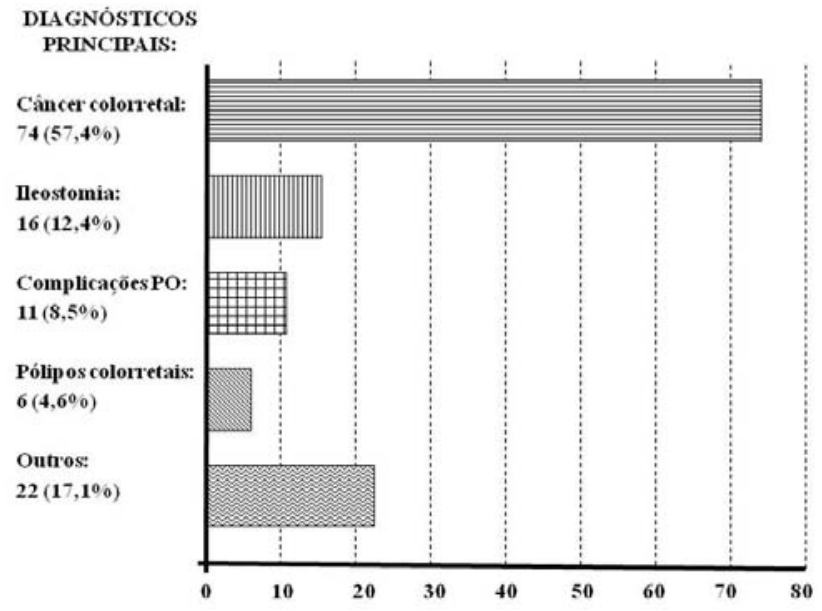

Figura 3 - Distribuição, por diagnósticos principais, de 129 pacientes submetidos a cirurgias êntero-colorretais, por dois médicos residentes em Coloproctologia como cirurgiões principais, no Programa de Pós-Graduação "sensu lato" do Grupo de Coloproctologia da SCBH e FCMMG. 
Rev bras Coloproct Julho/Setembro, 2010
Cirurgias Êntero-Colorretais - Abordagem Cirúrgica de 129 Pacientes do SUS no Programa de Pós-Graduação Sensu Lato em Coloproctologia Rodrigo Guimarães Oliveira e Cols.
Vol. 30 $\mathbf{N}^{\circ} 3$

Tabela 4 - Distribuição, em ordem decrescente, por cirurgias a que foram submetidos, de 129 pacientes abordados cirurgicamente, por dois médicos residentes em Coloproctologia como cirurgiões principais, no Programa de Pós-Graduação "sensu lato" do Grupo de Coloproctologia da SCBH e FCMMG em 2009.

\begin{tabular}{|c|c|c|}
\hline CIRURGIAS & $\mathbf{N}$ & $\%$ \\
\hline RSA-ACR ( 2 com ileostomia)* & 35 & 27,1 \\
\hline Com enterectomia e cistectomia & $\mathbf{2}$ & \\
\hline Com pan-histerectomia & 1 & \\
\hline HCD-AIT ( 2 com Ileostomia) & 20 & $\mathbf{1 5 , 5}$ \\
\hline Restabelecimento de trânsito (ileostomia) & 16 & 12,4 \\
\hline Laparotomia e colostomia (1 protetora de lesão anal) & 10 & 7,8 \\
\hline Hartmann & 7 & 5,4 \\
\hline CT-AIR ( 2 com ileostomia) & 6 & 4,7 \\
\hline Laparotomia e ileostomia (1 com cistorrafia) & 6 & 4,7 \\
\hline RSA-ACR-Duplo Grampeamento & 5 & $\mathbf{3 , 8}$ \\
\hline Duhamell & 5 & $\mathbf{3 , 8}$ \\
\hline Megacólon & 2 & \\
\hline Hartmann & 3 & \\
\hline HCE-ATR & 2 & 1,6 \\
\hline Re-abaixamento (1 com ileostomia) & 2 & 1,6 \\
\hline AAP-CD & 2 & 1,6 \\
\hline Enterectomia (1 com ileostomia) & 2 & 1,6 \\
\hline PCT-ID & $\mathbf{2}$ & 1,6 \\
\hline Laparorrafia & 2 & 1,6 \\
\hline Ressecção transanal & 2 & 1,6 \\
\hline Enterectomia e pan-histerectomia & 2 & 1,6 \\
\hline Reanastomose & 1 & $\mathbf{0 , 8}$ \\
\hline Retossigmoidectomia perineal & 1 & $\mathbf{0 , 8}$ \\
\hline Enterectomia e lise de aderências & 1 & $\mathbf{0 , 8}$ \\
\hline Total & 129 & 100,0 \\
\hline
\end{tabular}

Siglas usadas nesta e em todas as tabelas e figuras do texto: RSA$A C R=$ RetoSigmoidectomia Abdominal com Anastomose ColoRetal; HCD-AIT = HemiColectomia Direita com Anastomose Ileo-Transverso; RSA-ACR-DuGr: RSA-ACR com Duplo Grampeamento; $C T-A I R=$ Colectomia Total comAnastomose Íleo-Retal; HCE-ATR = HemiColectomia Esquerda com Anastomose Transverso-Retal; PCT-ID: Procto-Colectomia Total com Ileostomia Definitiva; AAP$C D$ : Amputação Abdômino-Perineal com Colostomia Definitiva.

anastomoses mecânicas com nove complicações $16,3 \%$ ) foram mas frequentes que as decorrentes de anastomoses manuais (21 anastomoses manuais com duas complicações - $(9,5 \%)$ (tabelas 6 e 8 e figura 7$)$. Houve 14 óbitos $(10,8 \%)$, sendo seis $(4,6 \%)$ devidos à sepse, quatro $(3,1 \%)$ devido a TEP e quatro $(3,1 \%)$ devido a falência múltipla de órgãos. Dos 14 pacientes que vieram a falecer, quatro $(3,1 \%)$ desenvolveram alguma complicação cirúrgica: dois desenvolveram

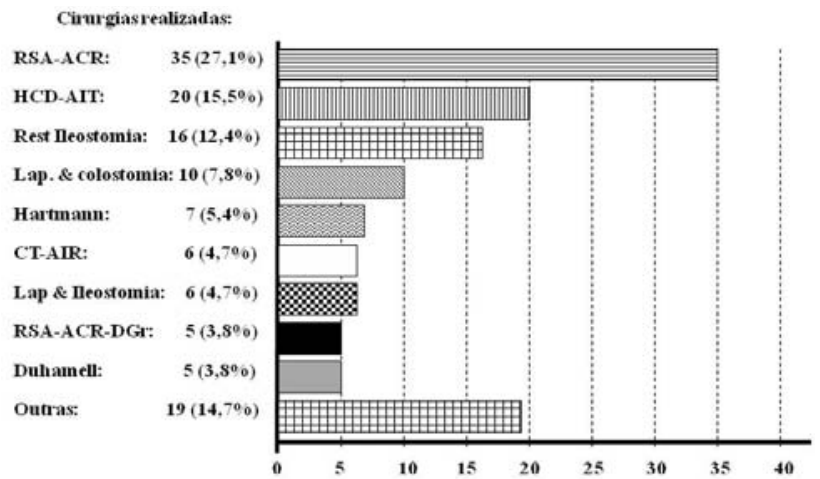

Figura 4 - Distribuição, por ordem decrescente e por cirurgias a que foram submetidos, de 129 pacientes abordados cirurgicamente, por dois médicos residentes em Coloproctologia como cirurgiões principais, no Programa de Pós-Graduação "sensu lato" do Grupo de Coloproctologia da SCBH e FCMMG, em 2009. 
Rev bras Coloproct Julho/Setembro, 2010
Cirurgias Êntero-Colorretais - Abordagem Cirúrgica de 129 Pacientes do SUS no Programa de Pós-Graduação Sensu Lato em Coloproctologia Rodrigo Guimarães Oliveira e Cols.
Vol. 30 $\mathbf{N}^{\circ} 3$

Tabela 5 - Distribuição, em ordem decrescente (separando-se os 76 que tiveram anastomoses intestinais $e$ os 53 sem anastomoses intestinais), por cirurgias a que foram submetidos, de 129 pacientes abordados cirurgicamente, por dois médicos residentes em Coloproctologia como cirurgiões principais, no Programa de Pós-Graduação "sensu lato” do Grupo de Coloproctologia da SCBH e FCMMG em 2009.

\begin{tabular}{|c|c|c|c|c|}
\hline CIRURGIAS & $\mathbf{N}$ & $\%$ & $\begin{array}{c}\text { Com } \\
\text { anast. }\end{array}$ & $\begin{array}{c}\text { Sem } \\
\text { anast. }\end{array}$ \\
\hline RSA-ACR ( 2 com ileostomia) & 35 & 27,1 & 35 & 0 \\
\hline Com enterect. e cistectomia & & & & \\
\hline Com pan-histerectomia & & & & \\
\hline HCD-AIT ( 2 com Ileostomia) & 20 & 15,5 & 20 & 0 \\
\hline CT-AIIR (2 com illeostomia) & 6 & 4,7 & 6 & 0 \\
\hline RSA-ACR-Duplo Grampeamento & 5 & 3,8 & 5 & 0 \\
\hline Duhamell & 5 & 3,8 & 5 & 0 \\
\hline Megacólon & & & & \\
\hline Hartmann & & & & \\
\hline HCE-ATR & 2 & 1,6 & 2 & 0 \\
\hline Re-abaixamento (1 com ileostomia) & 2 & 1,6 & 2 & 0 \\
\hline Reanastomose & 1 & 0,8 & 1 & 0 \\
\hline COM ANASTOMOSE & 76 & 58,9 & & \\
\hline Restabelecimento de trânsito (ileostomia) & 16 & 12,4 & $\mathbf{0}$ & 16 \\
\hline Laparot. e colostomia (1 protetora) & 10 & $\mathbf{7 , 8}$ & $\mathbf{0}$ & 10 \\
\hline Hartmann & 7 & $\mathbf{5 , 4}$ & $\mathbf{0}$ & 7 \\
\hline Laparot. e ileostomia (1 com cistorrafia) & 6 & 4,7 & $\mathbf{0}$ & 6 \\
\hline Amputação abdominoperineal & 2 & 1,6 & $\mathbf{0}$ & 2 \\
\hline Enterectomia (1 com ileostomia) & 2 & 1,6 & $\mathbf{0}$ & 2 \\
\hline PCT-IT & 2 & 1,6 & $\mathbf{0}$ & 2 \\
\hline Laparorrafia & 2 & 1,6 & $\mathbf{0}$ & 2 \\
\hline Ressecção transanal & 2 & 1,6 & $\mathbf{0}$ & 2 \\
\hline Enterectomia e pan-histerectomia & 2 & 1,6 & $\mathbf{0}$ & 2 \\
\hline Retossigmoidectomia perineal & 1 & 0,8 & $\mathbf{0}$ & 1 \\
\hline Enterectomia e lise de aderências & 1 & 0,8 & $\mathbf{0}$ & 1 \\
\hline SEM ANASTOMOSE & 53 & 41,1 & & \\
\hline Total & 129 & 100,0 & 76 & 53 \\
\hline
\end{tabular}

deiscência de anastomose; um, evisceração; e outro, trombose mesentérica. Os outros dez pacientes $(7,7 \%)$ faleceram sem complicação cirúrgica, mas por complicações decorrentes de suas co-morbidades: seis $(4,6 \%)$ por sepse, quatro $(3,1 \%)$ por TEP e quatro $(3,1 \%)$ por falência múltipla de órgãos. Dos quatro óbitos decorrentes de complicações cirúrgicas, dois foram dentre os 35 submetidos à retossigmoidectomia abdominal com anastomose colorretal, um dentre os 20 pacientes submetidos à hemicolectomia direita com anastomose ileo-transversa e um dentre os dois que se submeteram à reabaixamento (tabela 9).

Sumário dos resultados: A análise retrospectiva de 129 prontuários de pacientes do SUS, sub-
ANASTOMOSES:

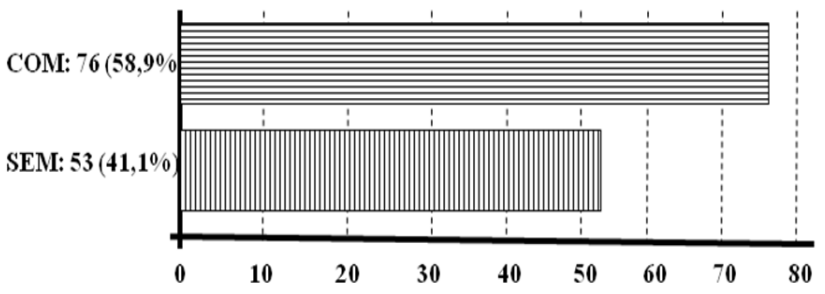

Figura 5 - Comparação entre os 76 em que houve anastomoses intestinais e os 53 sem anastomoses intestinais, entre os 129 pacientes abordados cirurgicamente, por dois médicos residentes em Coloproctologia como cirurgiões principais, no Programa de PósGraduação "sensu lato" do Grupo de Coloproctologia da SCBHe FCMMG em 2009. 
Rev bras Coloproct Julho/Setembro, 2010
Cirurgias Êntero-Colorretais - Abordagem Cirúrgica de 129 Pacientes do SUS no Programa de Pós-Graduação Sensu Lato em Coloproctologia Rodrigo Guimarães Oliveira e Cols.
Vol. 30

Tabela 6 - Distribuição, em ordem decrescente (separando-se os 76 que tiveram anastomoses intestinais dos 53 sem anastomoses intestinais), por cirurgias a que foram submetidos, analisando, separadamente, as complicações, os óbitos e as co-morbidades, de 129 pacientes abordados cirurgicamente, por dois médicos residentes em Coloproctologia como cirurgiões principais, no Programa de Pós-Graduação "sensu lato" do Grupo de Coloproctologia da SCBH e FCMMG.

\begin{tabular}{|c|c|c|c|c|c|c|c|}
\hline CIRURGIAS & $\mathbf{N}$ & $\%$ & $\begin{array}{c}\text { Com } \\
\text { anast. }\end{array}$ & $\begin{array}{c}\text { Sem } \\
\text { anast. }\end{array}$ & $\begin{array}{c}\text { Complic } \\
\text { ações }\end{array}$ & Òbitos & $\begin{array}{c}\text { Co- } \\
\text { morbid. }\end{array}$ \\
\hline RSA-ACR ( 2 com ileostomia) & 35 & 27,1 & 35 & 0 & 4 & 1 & 2 \\
\hline Com enterect. e cistectomia & 2 & & & & & & \\
\hline Com pan-histerectomia & 1 & & & & & & \\
\hline HCD-AIT (2 com Ileostomia) & 20 & 15,5 & 20 & 0 & 3 & 2 & 2 \\
\hline CT-AIR (2 com illeostomia) & 6 & 4,7 & 6 & 0 & 3 & 0 & 1 \\
\hline RSA-ACR-Duplo Grampeamento & 5 & 3,8 & 5 & 0 & 1 & 0 & 1 \\
\hline Duhamell & 5 & 3,8 & 5 & 0 & 0 & 0 & 0 \\
\hline Megacólon & 2 & & & & & & \\
\hline Hartmann & 3 & & & & & & \\
\hline HCE-ATR & 2 & 1,6 & 2 & 0 & 0 & 0 & 0 \\
\hline Re-abaixamento (1 com ileostomia) & 2 & 1,6 & 2 & 0 & 0 & 1 & 2 \\
\hline Reanastomose & 1 & 0,8 & 1 & 0 & 0 & 0 & 0 \\
\hline COM ANASTOMOSE & 76 & 58,9 & 76 & 0 & 11 & 4 & 8 \\
\hline Restabelecimento de trânsito (ileostomia) & 16 & 12,4 & $\mathbf{0}$ & 16 & 1 & $\mathbf{0}$ & $\mathbf{0}$ \\
\hline Laparot. e colostomia (1 protetora) & 10 & $\mathbf{7 , 8}$ & $\mathbf{0}$ & 10 & 2 & 4 & 5 \\
\hline Hartmann & 7 & $\mathbf{5 , 4}$ & $\mathbf{0}$ & 7 & 3 & 3 & 3 \\
\hline Laparot. e ileostomia (1 com cistorrafia) & 6 & 4,7 & $\mathbf{0}$ & 6 & $\mathbf{0}$ & 1 & 1 \\
\hline Amputação abdominoperineal & 2 & 1,6 & $\mathbf{0}$ & 2 & $\mathbf{0}$ & $\mathbf{0}$ & 1 \\
\hline Enterectomia (1 com ileostomia) & 2 & 1,6 & $\mathbf{0}$ & 2 & $\mathbf{0}$ & $\mathbf{0}$ & $\mathbf{0}$ \\
\hline PCT-IT & 2 & 1,6 & $\mathbf{0}$ & 2 & $\mathbf{0}$ & 1 & 1 \\
\hline Laparorrafia & 2 & 1,6 & $\mathbf{0}$ & 2 & $\mathbf{0}$ & $\mathbf{0}$ & $\mathbf{0}$ \\
\hline Ressecção transanal & 2 & 1,6 & $\mathbf{0}$ & 2 & $\mathbf{0}$ & $\mathbf{0}$ & $\mathbf{0}$ \\
\hline Enterectomia e pan-histerectomia & 2 & 1,6 & $\mathbf{0}$ & 2 & $\mathbf{0}$ & 1 & 1 \\
\hline Retossigmoidectomia perineal & 1 & 0,8 & $\mathbf{0}$ & 1 & $\mathbf{0}$ & $\mathbf{0}$ & $\mathbf{0}$ \\
\hline Enterectomia e lise de aderências & 1 & 0,8 & $\mathbf{0}$ & 1 & $\mathbf{0}$ & $\mathbf{0}$ & $\mathbf{0}$ \\
\hline SEM ANASTOMOSE & 53 & 41,1 & $\mathbf{0}$ & 53 & 6 & 10 & 12 \\
\hline TOTAL & 129 & 100,0 & 76 & 53 & 17 & 14 & 20 \\
\hline
\end{tabular}

metidos a várias cirurgias êntero-colorretais, pelo Residente R2 supervisionado e auxiliado por preceptores, permitiu a observação dos seguintes resultados:

1. A média etária dos pacientes foi 56,9 anos, com extremos de 25 e 87 anos, sendo as sexta e sétima décadas a mais representativa, respectivamente com $25,6 \%$ e $24,8 \%$, totalizando $50,4 \%$ dos 55 pacientes $(\mathrm{p}<0,05)$.

2. Dos 129 pacientes, $51,2 \%$ eram sexo feminino $(51,2 \%)(\mathrm{p}>0,05)$.

3. A entidade nosológica mais comum foi o câncer colorretal (74 casos; $57,4 \%$ ), seguindo as ileostomias (16 casos; $12,4 \%)$ e as complicações cirúrgicas (11 casos; 8,5\%).

4. As cirurgias mais realizadas foram as retossigmoidectomia com anastomose colorretal (35 casos; 27,1\%), as hemicolectomias direitas com anastomose ileo-transverso (20 casos; $15,5 \%)$ e o

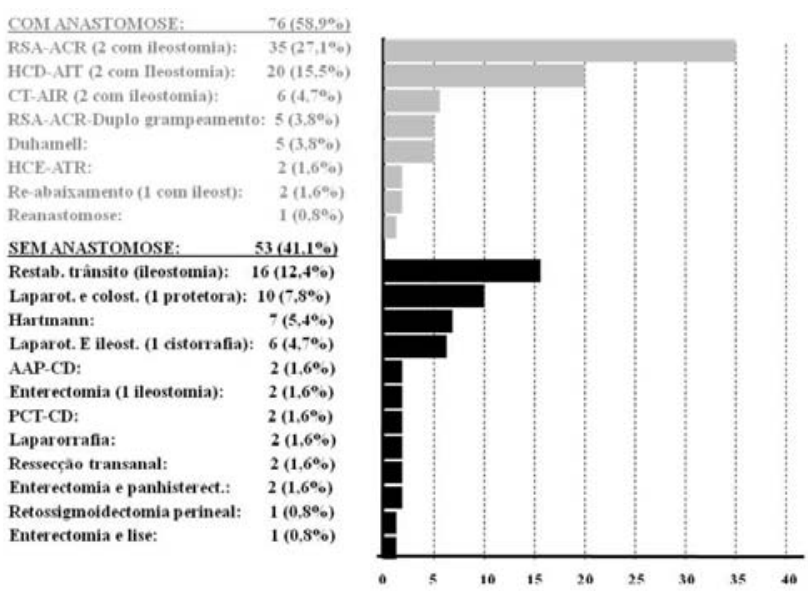

Figura 6 - Distribuição, em ordem decrescente (separando-se os 76 que tiveram anastomoses intestinais e os 53 sem anastomoses intestinais), por cirurgias a que foram submetidos, de 129 pacientes abordados cirurgicamente, por dois médicos residentes em Coloproctologia como cirurgiões principais, no Programa de PósGraduação "sensu lato" do Grupo de Coloproctologia da SCBH e FCMMG em 2009. 
Rev bras Coloproct Julho/Setembro, 2010
Cirurgias Êntero-Colorretais - Abordagem Cirúrgica de 129 Pacientes do SUS no Programa de Pós-Graduação Sensu Lato em Coloproctologia Rodrigo Guimarães Oliveira e Cols.
Vol. 30

Tabela 7 - Distribuição, em ordem decrescente (separando-se os 76 que tiveram anastomoses intestinais dos 53 sem anastomoses intestinais), por cirurgias a que foram submetidos, analisando a incidência de co-morbidades, de 129 pacientes abordados cirurgicamente, por dois médicos residentes em Coloproctologia como cirurgiões principais, no Programa de Pós-Graduação "sensu lato" do Grupo de Coloproctologia da SCBH e FCMMG.

\begin{tabular}{l|c|c|c|l|}
\hline \multicolumn{1}{|c|}{ CIRURGIAS } & $\mathbf{N}$ & $\mathbf{\%}$ & $\begin{array}{c}\text { Co- } \\
\text { morbid. }\end{array}$ & Co-morbidades \\
\hline RSA-ACR (2 com ileostomia) & 35 & 27,1 & 2 & Obesidade; caquexia \\
\hline HCD-AIT (2 com Illeostomia) & 20 & 15,5 & 2 & Caquexia; caquexia \\
\hline CT-AIR (2 com illeostomia) & 6 & 4,7 & 1 & Diabete e anemia \\
\hline RSA-ACR-Duplo Grampeamento & 5 & 3,8 & 1 & Hipertensão arterial e caquexia \\
\hline Duhamell & 5 & 3,8 & 0 & \\
\hline HCE-ATR & 2 & 1,6 & 0 & \\
\hline Re-abaixamento (1 com ileostomia) & 2 & 1,6 & 2 & Hipertensão arterial; bronquiectasia \\
\hline Reanastomose & 1 & 0,8 & 0 & \\
\hline COM ANASTOMOSE & 76 & 58,9 & 8 & \\
\hline Restabelecimento de trânsito (ileostomia) & $\mathbf{1 6}$ & $\mathbf{1 2 , 4}$ & $\mathbf{0}$ & \\
\hline Laparot. e colostomia (1 protetora) & $\mathbf{1 0}$ & $\mathbf{7 , 8}$ & $\mathbf{5}$ & Todos caquexia \\
\hline Hartmann & $\mathbf{7}$ & $\mathbf{5 , 4}$ & $\mathbf{3}$ & Caquexia; insuf. Cardíaca; caquexia \\
\hline Laparot. e ileostomia (1 com cistorrafia) & $\mathbf{6}$ & $\mathbf{4 , 7}$ & $\mathbf{1}$ & Caquexia \\
\hline Amputação abdominoperineal & $\mathbf{2}$ & $\mathbf{1 , 6}$ & $\mathbf{1}$ & Hipertensão arterial e insuf. cardíaca \\
\hline Enterectomia (1 com ileostomia) & $\mathbf{2}$ & $\mathbf{1 , 6}$ & $\mathbf{0}$ & \\
\hline PCT-IT & $\mathbf{2}$ & $\mathbf{1 , 6}$ & $\mathbf{1}$ & Obstrução intestinal \\
\hline Laparorrafia & $\mathbf{2}$ & $\mathbf{1 , 6}$ & $\mathbf{0}$ & \\
\hline Ressecção transanal & $\mathbf{2}$ & $\mathbf{1 , 6}$ & $\mathbf{0}$ & \\
\hline Enterectomia e pan-histerectomia & $\mathbf{2}$ & $\mathbf{1 , 6}$ & $\mathbf{1}$ & Caquexia \\
\hline Retossigmoidectomia perineal & $\mathbf{1}$ & $\mathbf{0 , 8}$ & $\mathbf{0}$ & \\
\hline Enterectomia e lise de aderências & $\mathbf{1}$ & $\mathbf{0 , 8}$ & $\mathbf{0}$ & \\
\hline SEM ANASTOMOSE & $\mathbf{5 3}$ & $\mathbf{4 1 , 1}$ & $\mathbf{1 2}$ & \\
\hline Total & $\mathbf{1 2 9}$ & $\mathbf{1 0 0 , 0}$ & $\mathbf{2 0}$ & \\
\hline
\end{tabular}

restabelecimento de trânsito intestinal de ileostomia (16 casos; $12,4 \%$ ).

5. Das 129 cirurgias $53(41,1 \%)$ não envolveram anastomoses e $76(58,9 \%)$ envolveram ressecções intestinais e anastomoses.

6. Houve oito co-morbidades $(6,2 \%)$, sendo a caquexia (três casos) a mais comum.

7. Houve 17 complicações $(13,2 \%)$, 11 envolvendo as 76 ressecções com anastomose $(14,5 \%)$ e seis as ressecções sem anastomoses $(11,3 \%)$.

8. As complicações mais comuns entre as 11 provenientes de ressecções e anastomoses foram as deiscências (sete; 9,2\%).

9. As anastomoses mecânicas (55) complicaram mais $(16,3 \%)$ que as manuais (21) $(9,5 \%)$.

10. Houve 14 óbitos $(10,8 \%)$, sendo seis $(4,6 \%)$ devidos à sepse, quatro $(3,1 \%)$ devido a TEP e quatro $(3,1 \%)$ devido a falência múltipla de órgãos.

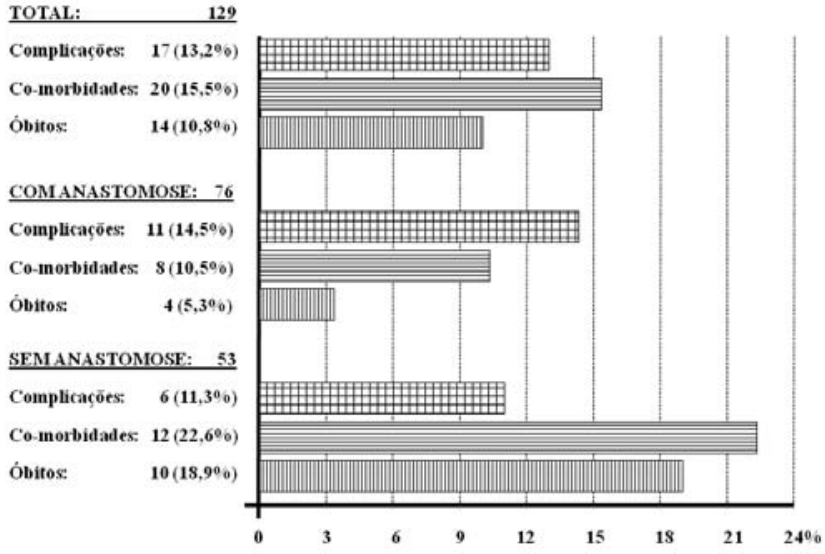

Figura 7 - Distribuição, em ordem decrescente (separando-se os 76 que tiveram anastomoses intestinais dos 53 sem anastomoses intestinais), por cirurgias a que foram submetidos, analisando, separadamente, as complicações, os óbitos e as co-morbidades, de 129 pacientes abordados cirurgicamente, por dois médicos residentes em Coloproctologia como cirurgiões principais, no Programa de Pós-Graduação "sensu lato" do Grupo de Coloproctologia da SCBH e FCMMG. 
Rev bras Coloproct Julho/Setembro, 2010
Cirurgias Êntero-Colorretais - Abordagem Cirúrgica de 129 Pacientes do SUS no Programa de Pós-Graduação Sensu Lato em Coloproctologia Rodrigo Guimarães Oliveira e Cols.
Vol. 30

Tabela 8 - Distribuição, em ordem decrescente (separando-se os 76 que tiveram anastomoses intestinais dos 53 sem anastomoses intestinais), por cirurgias a que foram submetidos, analisando a incidência de complicações, de 129 pacientes abordados cirurgicamente, por dois médicos residentes em Coloproctologia como cirurgiões principais, no Programa de Pós-Graduação "sensu lato" do Grupo de Coloproctologia da SCBH e FCMMG.

\begin{tabular}{l|c|c|c|l|}
\hline \multicolumn{1}{|c|}{ CIRURGIAS } & N & \% & Complic. & \multicolumn{1}{c|}{ Complicações } \\
\hline RSA-ACR (2 com illeostomia) & 35 & 27,1 & 4 & Deiscência anast. (3); fístulla vaginal \\
\hline HCD-AIT (2 com Illeostomia) & 20 & 15,5 & 3 & Deiscência (2);TEP \\
\hline CT-AIR (2 com illeostomia) & 6 & 4,7 & 3 & Trombose mesent.; deiscência; abscesso \\
\hline RSA-ACR-Duplo Grampeamento & 5 & 3,8 & 1 & Deiscência \\
\hline Duhamell & 5 & 3,8 & 0 & \\
\hline HCE-ATR & 2 & 1,6 & 0 & \\
\hline Re-abaixamento (1 com illeostomia) & 2 & 1,6 & 0 & \\
\hline Reanastomose & 1 & 0,8 & 0 & \\
\hline COM ANASTOMOSE & 76 & 58,9 & 11 & \\
\hline Restabelecimento de trânsito (ileostomia) & $\mathbf{1 6}$ & $\mathbf{1 2 , 4}$ & $\mathbf{1}$ & Fístula anastomótica \\
\hline Laparot. e colostomia (1 protetora) & $\mathbf{1 0}$ & $\mathbf{7 , 8}$ & $\mathbf{2}$ & Fístula retovesical; evisceração \\
\hline Hartmann & $\mathbf{7}$ & $\mathbf{5 , 4}$ & $\mathbf{3}$ & Evisceração;necrose de coto; sepse \\
\hline Laparot. e ileostomia (1 com cistorrafia) & $\mathbf{6}$ & $\mathbf{4 , 7}$ & $\mathbf{0}$ & \\
\hline Amputação abdominoperineal & $\mathbf{2}$ & $\mathbf{1 , 6}$ & $\mathbf{0}$ & \\
\hline Enterectomia (1 com ileostomia) & $\mathbf{2}$ & $\mathbf{1 , 6}$ & $\mathbf{0}$ & \\
\hline PCT-IT & $\mathbf{2}$ & $\mathbf{1 , 6}$ & $\mathbf{0}$ & \\
\hline Laparorrafia & $\mathbf{2}$ & $\mathbf{1 , 6}$ & $\mathbf{0}$ & \\
\hline Ressecção transanal & $\mathbf{2}$ & $\mathbf{1 , 6}$ & $\mathbf{0}$ & \\
\hline Enterectomia e pan-histerectomia & $\mathbf{2}$ & $\mathbf{1 , 6}$ & $\mathbf{0}$ & \\
\hline Retossigmoidectomia perineal & $\mathbf{1}$ & $\mathbf{0 , 8}$ & $\mathbf{0}$ & \\
\hline Enterectomia e lise de aderências & $\mathbf{1}$ & $\mathbf{0 , 8}$ & $\mathbf{0}$ & \\
\hline SEM ANASTOMOSE & $\mathbf{5 3}$ & $\mathbf{4 1 , 1}$ & $\mathbf{6}$ & \\
\hline Total & $\mathbf{1 2 9}$ & $\mathbf{1 0 0 , 0}$ & $\mathbf{1 7}$ & \\
\hline
\end{tabular}

11. Dos 14 óbitos, quatro $(3,1 \%)$ foram decorrentes de complicações cirúrgicas e dez $(7,7 \%)$ foram decorrentes de co-morbidades.

\section{DISCUSSÃO}

A especialização médica em coloproctologia é bastante diversificada em todo o território nacional. Critérios mínimos são estabelecidos tanto pelas Comissões de Residência Médica estaduais quanto pelos serviços de coloproctologia credenciados pela Sociedade Brasileira de Coloproctologia, na tentativa de padronizar os treinamentos médicos.

De acordo com as exigências básicas para obtenção do título de especialista em coloproctologia pela Sociedade Brasileira de Coloproctologia, exigemse 15 cirurgias colorretais durante o programa de treinamento médico. A estatística apresentada no trabalho mostra claramente que a formação oferecida pela Santa Casa de Misericórdia de Belo Horizonte supera, de forma grandiosa, esta exigência mínima, sendo que cada residente realizou, no mínimo, 64 cirurgias colorretais, com uma grande variedade de procedimentos conforme demonstrado na tabela 4 e figura 4 .

A idade média dos pacientes foi de 56,9 anos, porém com uma maior frequência nos pacientes na sexta e sétima década de vida. Este dado é condizente com dados da literatura, que demonstram um aumento das afecções colorretais, principalmente quanto a incidência de câncer colorretal, com o aumento da faixa etária ${ }^{1}$.

Setenta e quatro casos $(57,4 \%)$ das cirurgias realizadas objetivaram o tratamento de neoplasias colorretais. Nota-se uma distribuição topográfica semelhante a dados da literatura, com uma maior prevalência de neoplasias de reto e cólon sigmóide ${ }^{1}$. 
Rev bras Coloproct Julho/Setembro, 2010
Cirurgias Êntero-Colorretais - Abordagem Cirúrgica de 129 Pacientes do SUS no Programa de Pós-Graduação Sensu Lato em Coloproctologia Rodrigo Guimarães Oliveira e Cols.
Vol. 30

Tabela 9 - Distribuição, em ordem decrescente (separando-se os 76 que tiveram anastomoses intestinais dos 53 sem anastomoses intestinais), por cirurgias a que foram submetidos, analisando a incidência de comorbidade relacionada à incidência de óbitos, de 129 pacientes abordados cirurgicamente, por dois médicos residentes em Coloproctologia como cirurgiões principais, no Programa de Pós-Graduação "sensu lato" do Grupo de Coloproctologia da SCBH e FCMMG.

\begin{tabular}{|c|c|c|c|c|}
\hline CIRURGLAS & $\mathbf{N}$ & $\%$ & CO-MORBIDADES & ÓBITOS \\
\hline RSA-ACR ( 2 com ileostomia) & 35 & 27,1 & $\begin{array}{l}\text { Obesidlade } \\
\text { Caquexia }\end{array}$ & Sepse \\
\hline HCD-AIT (2 com Ileostomia) & 20 & 15,5 & $\begin{array}{l}\text { Caquexia } \\
\text { Caquexia }\end{array}$ & $\begin{array}{l}\text { TEP } \\
\text { TEP }\end{array}$ \\
\hline CT-AIR (2 com ileostomia) & 6 & 4.7 & DM e anemia & \\
\hline RSA-ACR-Duplo Grampeamento & 5 & 3,8 & HAS e caquexia & \\
\hline Duhamell & 5 & 3,8 & & \\
\hline HCE-ATR & 2 & 1,6 & & \\
\hline Re-abaixamento ( 1 com ileostomia) & 2 & 1,6 & $\begin{array}{l}\text { HAS; } \\
\text { Bronquiectasia }\end{array}$ & Sepse \\
\hline Reanastomose & 1 & 0,8 & & \\
\hline COMANASTOMOSE & 76 & 58.9 & 8 & 4 \\
\hline Restabelecimento de trânsito (ileostomia) & 16 & 12,4 & & \\
\hline Laparot. e colostomia (1 protetora) & 10 & 7,8 & $\begin{array}{l}\text { Caquexia e OIM } \\
\text { Caquexia } \\
\text { Caquexia } \\
\text { Caquexia } \\
\text { Caquexia } \\
\end{array}$ & \begin{tabular}{|l|} 
Sepse \\
TEP \\
Complic. cir. (deiscência) \\
Sepse
\end{tabular} \\
\hline Hartmann & 7 & 5,4 & $\begin{array}{l}\text { Caquexia } \\
\text { Caquexia e ins. resp. } \\
\text { Caquexia e ins. card. }\end{array}$ & $\begin{array}{l}\text { Falência múltipla } \\
\text { Falência múltipla } \\
\text { Sepse }\end{array}$ \\
\hline Laparot. e ileostomia (1 com cistorrafia) & 6 & 4,7 & Caquexia & TEP \\
\hline Amputação abdominoperineal & 2 & 1,6 & HAS e ins. Card. & \\
\hline Enterectomia (1 com ileostomia) & 2 & 1,6 & & \\
\hline PCT-IT & 2 & 1,6 & OIM e HAS & Falência múltipla \\
\hline Laparorrafia & 2 & 1,6 & & \\
\hline Ressecç̃o transanal & 2 & 1,6 & & \\
\hline Enterectomia e pan-histerectomia & 2 & 1,6 & Caquexia & Sepse \\
\hline Retossigmoidectomia perineal & 1 & 0,8 & & \\
\hline Enterectomia e lise de aderências & 1 & 0,8 & & \\
\hline SEMANASTOMOSE & 53 & $+1,1$ & 12 & 10 \\
\hline Total & 129 & 100,0 & 20 & 14 \\
\hline
\end{tabular}

Das 129 cirurgias realizadas, 53 (41,1\% dos casos) envolveram a realização de ostomias, sejam temporárias ou definitivas. A justificativa para tal estatística baseia-se no tratamento de neoplasias colorretais avançadas e inoperáveis, com objetivo de descompressão colônica e desvio de trânsito; pacientes graves, sem condições para a realização de anastomoses colônicas; desnutrição avançada, peritonite fecal. Orkin et al estimaram que 750.000 americanos sejam ostomizados, e que sejam realizadas aproximadamente 75.000 novas ostomias nos Estados Unidos anualmente ${ }^{2}$. No serviço de Coloproctologia da Santa Casa de Misericórdia de Belo Horizonte, tenta-se ao máximo preservar o paciente da construção de ostomias, salvo os casos onde sua indicação é formal.

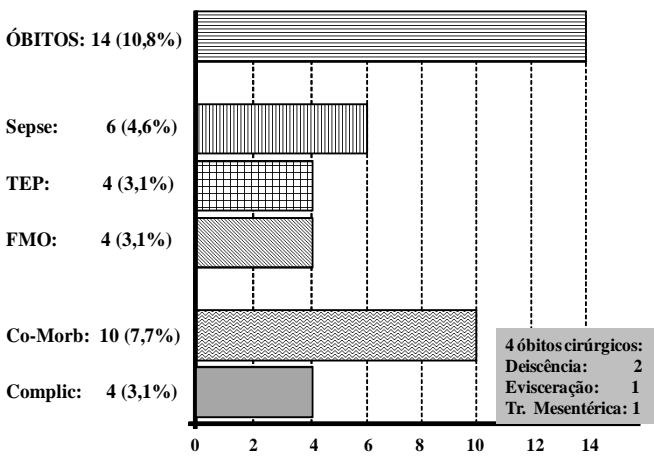

Figura 9 - Distribuição, em ordem decrescente (separando-se os 76 que tiveram anastomoses intestinais dos 53 sem anastomoses intestinais), por cirurgias a que foram submetidos, analisando a incidência de comorbidade relacionada à incidência de óbitos, de 129 pacientes abordados cirurgicamente, por dois médicos residentes em Coloproctologia como cirurgiões principais, no Programa de Pós-Graduação "sensu lato" do Grupo de Coloproctologia da SCBH e FCMMG. 
Rev bras Coloproct Julho/Setembro, 2010
Cirurgias Êntero-Colorretais - Abordagem Cirúrgica de 129 Pacientes do SUS no Programa de Pós-Graduação Sensu Lato em Coloproctologia Rodrigo Guimarães Oliveira e Cols.
Vol. 30 $\mathbf{N}^{\circ} 3$
Dentre as complicações relacionadas à cirurgia, a mais comum foi a deiscência de anastomose $(9$ casos dentre as 76 cirurgias que combinaram ressecção e anastomose - 9,2\% dos casos), sendo mais comum nos casos de anastomoses baixas realizadas em retossigmoidectomias abdominais (11\% dos casos). Dados comparativos da literatura mostram uma incidência de $6,7 \%$, contudo variando com a topografia da anastomose (8\% para anastomoses baixas e $1 \%$ para anastomoses altas $)^{3,4}$. Além disso, Hayanga et al demonstraram que a taxa de complicações relacionadas a cirurgias colorretais são maiores em hospitais-escola ${ }^{5}$, o que condiz com a realidade do serviço de coloproctologia da Santa Casa de Belo Horizonte.
A taxa de mortalidade relacionada a complicações cirúrgicas foi de 3,1\% (quatro de 129 casos). Dados da literatura apresentam taxas médias de 2,2\% dos $\operatorname{casos}^{3}$.

\section{CONCLUSÃO}

A análise de 129 pacientes do SUS atendidos em regime hospitalar visando cirurgias abdominais êntero-colorretais permitiu verificar que a o diagnóstico de câncer colorretal foi o mais comum, que as cirurgias mais realizadas foram as retossigmoidecomias abdominais com anastomose colorretal, sendo a morbimortalidade elevada em decorrência do estado nutricional e das co-morbidades apresentadas pelos pacientes.

ABSTRACT: In the framework of postgraduate Coloproctology for the year 2009, the two graduate students conducted the second year as principal surgeons, 129 major surgeries, always assisted effectively by one or two tutors. All surgeries were performed on public patients in Santa Casa de Belo Horizonte, with absolute presence of members of the Coloproctology Unit of Santa Casa School of Medical Sciences of Minas Gerais (GCP-CBHS-FCMMG). A retrospective analysis of 129 medical records was carried out, allowing several important observations. The average age of patients was 56.9 years, with extremes of 25 and 87 years, while the sixth and seventh decades the most representative, with respectively $25.6 \%$ and $24.8 \%$ to $50.4 \%$ of 55 patients (p<0.05). Most of the 129 patients were female $(51.2 \%)(p>0.05)$. The most common nosological entity was colorectal cancer $(\mathbf{7 4}$ cases, $57.4 \%)$, following the ileostomies ( 16 cases, $\mathbf{1 2 . 4 \%}$ ) and surgical complications of previous surgeries (11 cases, $8.5 \%$ ). The most commonly performed procedures were abdominal rectosigmoidectomy with colorectal anastomosis (35 cases, $27.1 \%$ ), the right hemicolectomy with ileo-transverse anastomosis (20 cases, $15.5 \%)$ and the resumption of intestinal transit of ileostomy $(16$ cases, $12,4 \%)$. Of 129 surgeries $53(41.1 \%)$ did not involve anastomosis and $76(58.9 \%)$ involved intestinal resection and anastomosis. There were eight co-morbidities $(6.2 \%)$ and cachexia (three cases) the most common. There were 17 complications (13.2\%), 11 involving the 76 resections with anastomosis $(14.5 \%)$ and six resections without anastomosis $(11.3 \%)$. The most common complications among the 11 patients from resection and anastomosis were dehiscence (seven, 9.2\%). The mechanical anastomosis (55) developed more complications $(16.3 \%)$ than handmade anastomosis $(21)(9.5 \%)$. There were 14 deaths $(10.8 \%)$, six $(4.6 \%)$ due to sepsis, four (3.1\%) due to pulmonary thromboembolic disease and four (3.1\%) due to multiple organ failure. Of the 14 deaths, four (3.1\%) were due to surgical complications and ten $(7.7 \%)$ were due to co-morbidities.

Key words: Colorectal surgery; abdominal surgery; review; public patients; postgraduate program.

\section{BIBLIOGRAFIA}

1. Baxter N, Guillem J. Colorectal Cancer: Epidemiology, Etiology, and Molecular Basis. In: The ASCRS Textbook of Colon and Rectal Surgery 2008:335-352. Springer.

2. Orkin B, Cataldo P. Intestinal Stomas. In: The ASCRS Textbook of Colon and Rectal Surgery 2008:622-642. Springer.

3. Basilico V, Griffa B, Castiglione N, Giacci F, Zanardo M, Griffa A. Anastomotic fistulas after colorectal resection for carcinoma: incidence and treatment in our recent experience. Minerva Chir. 2006 Oct;61(5):373-80.

4. Dietz D, Bailey H. Postoperative Complications. In: The ASCRS Textbook of Colon and Rectal Surgery 2008:141-155. Springer.
5. Hayanga AJ, Mukherjee D, Chang D, Kaiser H, Lee T, Gearhart S, Ahuja N, Freischlag J. Teaching hospital status and operative mortality in the United States: tipping point in the volumeoutcome relationship following colon resections? Arch Surg. 2010 Apr;145(4):346-50.

Endereço para correspondência: GERALDO MAGELA GOMES DA CRUZ

Rua Rio de Janeiro, 2017 / 1401 - Lourdes

Belo Horizonte - MG

CEP: 30130-042 